\title{
LEER PARA COMPRENDER: DIEZ AÑOS DE ESTUDIOS SOBRE LOS PROCESOS LECTORES
}

\section{READ AND COMPREHEND: A DECADE OF STUDIES ON THE READING PROCESSES}

\author{
Ariel Cuadro \\ Coordinador del Número Monográfico \\ Universidad Católica del Uruguay, Uruguay
}

\begin{abstract}
RESUMEN
La lectura constituye en la actualidad una de las competencia más importante para el desarrollo humano. En la introducción de este monográfico se señalan las líneas de trabajo que hemos seguido a lo largo de estos diez años de estudios en psicología de lectura y se presentan investigaciones realizadas en nuestro medio y otras, por colegas que nos han visitado a los largo de todos estos años. Sus aportes resultan fundamentales para la comprensión de los procesos de lectura desde una perspectiva cognitiva, brindándonos herramientas que nos permitan educar lectores estratégicos capaces de integrarse en la sociedad del conocimiento.
\end{abstract}

Palabras clave: Psicología de la lectura, investigación, revisión

\begin{abstract}
The reading process constitutes at present day one of most important competencies involved in human development. Theintroduction of this monographicissue brings to light the research lines we have been working on throughout ten years of studies in psychology of the reading processes. Investigations conducted both locally and internationally are presented. Contributions emerged from these studies turn out to be fundamental for the understanding of the reading processes from a cognitive perspective, providing tools that enable us to instruct strategic readers who are capable of being part of the knowledge society.
\end{abstract}

Key words: Psychology of reading, research, review

A finales de los años noventa iniciamos en la Facultad de Psicología, de la Universidad Católica del Uruguay, los estudios sobre la lectura, su aprendizaje y sus dificultades. Nos impulsaba la relevancia que el aprendizaje de la lectura tiene para la educación del niño y particularmente para su desarrollo.

Contábamos con el apoyo y la orientación del Prof. Javier Marín de la Universidad de Murcia, quien a su vez estaba trabajando con destacados especialistas internacionales en la temática.

La lectura es una actividad cognitiva compleja que resulta fundamental para la adaptación e integración de las personas a nuestra sociedad del conocimiento. Los aportes de la Psicología Cognitiva nos han permitido reconocer los procesos cognitivos subyacentes a la actividad lectora, y comprender la lectura eficiente, la adquisición de la lectura y particularmente los trastornos en lectura que conllevan frecuentemente al fracaso escolar.

Al iniciar nuestros trabajos nos encontramos que en Uruguay, así como en otros países de la región, no habían criterios precisos al momento definir el concepto de lectura, sus trastornos, ni técnicas actualizadas para diagnosticarlos. Nos propusimos entonces poner en marcha la validación de un conjunto de pruebas que permitieran en nuestro medio evaluar el nivel lector y los procesos implicados en la lectura. De este modo en estos años hemos estandarizado test y desarrollado diversas tareas con ese fin (Cuadro, Costa, Trías y Ponce de León, 2009). 
En el estudio de los procesos lectores la identificación de los mecanismos de reconocimiento de palabras ha resultado esencial para comprender la lectura eficiente así como para explicar las dificultades que se presentan durante su adquisición. Los aportes de los trabajos que estudian dichos procesos en lengua española resultan esenciales para entender las particularidades de los lectores hispanohablantes (Marin, Cuadro, Pagán 2007). Es así que hemos caracterizado a los lectores retrasados, identificando subtipos de disléxicos uruguayos determinado su incidencia y los perfiles cognitivos que tienen; los que a su vez venimos comparado con otros estudios en español y en otros sistemas ortográficos (Cuadro, Marin, 2007; Marín, Aveledo, Pagán y Cuadro, 2007; Marín, Pagán, Cuadro y Aveledo, 2007; Cuadro 2006). Los resultados obtenidos nos permiten analizar y evaluar variables que inciden en el aprendizaje lector: conciencia fonológica, velocidad de nominación, vocabulario (Cuadro, Ilunday y Puig, 2009; Cuadro, Trías, 2008; Carballo, Cuadro, 2007), así como, desarrollar programas de intervención para mejorar la habilidad lectora considerando las diversas realidades socioculturales del país (Cuadro, Trias, Castro, 2007). Finalmente el interés que la investigación cognitiva ha tenido por la lectura, más específicamente por los procesos psicológicos que involucra, también nos ha posibilitado conocer más sobre el acceso lector en sistemas de escritura diferentes, así como en personas con deficiencias auditivas o visuales. Lo que nos ha llevado a realizar trabajos con lectores ciegos y sordos, los que han incluido el desarrollo de pruebas de evaluación específicas (Cuadro, Almansa; 2008; Cuadro Piñeyro, 2008).

El desarrollo de nuestro trabajo se ha realizado y se realiza con la colaboración de investigadores relevantes en el área, como lo son Jesús Alegría, Marisol Carrillo, Javier Marín, Luis Bravo Valdivieso, Aldo Ferreres, Valeria Abusambra y otros, que a lo largo de estos años nos han visitado, animado, orientado y enseñado con sus trabajos.

El siguiente monográfico quiere ser, a partir de la invitación de participar con trabajos a los autores con los que compartimos estos temas, una expresión más de la relevancia que los estudios sobre la lectura tienen en el español, para el mejoramiento de las prácticas educativas, el diseño de políticas curriculares y la formación de docentes.

El Comité Científico de la Revista tuvo la ardua tarea de evaluar y dictaminar sobre una gran cantidad de colaboraciones, de entre las cuales se han seleccionado las diez que componen este Número Monográfico.

El primer artículo original, a cargo de del Dres. Carrillo y Alegría presenta los resultados sobre la existencia de un déficit del mecanismo de lectura fonológico y así mismo del mecanismo léxico ortográfico en la dislexia de evolución. Sus aportes enriquecen la mirada sobre estos procesos y generan múltiples caminos de trabajo futuro.

Por su parte Balbi, Cuadro y Trias, basados en el modelo simple de lectura propuesto por Gough y Tunmer (1986), centran su estudio en la relación existente entre la comprensión de textos y el reconocimiento de palabras. El objetivo de su investigación radica en precisar la relativa independencia que habría al correlacionar habilidades de procesamiento léxico-semántico y fluidez, con la comprensión de textos, en sujetos de $4^{\circ}, 5^{\circ}$ y $6^{\circ}$ grado escolar.

Los Profesores Ferrés y Lopez, de la Universidad de Buenos Aires, presentan una revisión de casos de alexia en hispanohablantes. Si bien se ha cuestionado la existencia de cuadros de alexia fonológica, de superficie y profunda en la población hispana, debido a la utilización de sistemas de escritura "transparentes"; y la pertinencia de los modelos de doble ruta para explicar dicho trastorno; la investigación llevada a cabo aporta evidencia empírica en pos de estos asuntos tan esenciales.

En el artículo que sigue, nuestro equipo (Trias, Cuadro y Costa), se enfoca en el estudio longitudinal del impacto del entrenamiento en conciencia fonémica en la adquisición del lenguaje escrito, a partir de la evaluación de un programa de intervención. Los resultados planteados en este trabajo, dejan abierto todo un campo de trabajo, que cada vez recibe mayor demanda para los profesionales de la especialidad.

Meneghetti, Carretti, De Beni, Cornoldi y Abusamra, señalan los aportes de la comprensión de textos desde una perspectiva componencial, 
a nivel de mejoramiento, buscando verificar la eficacia de un entrenamiento de la habilidad para individualizar personajes, lugar y tiempo Se proponen tres evaluaciones -según momento del proceso de intervención- y un programa de tratamiento - Nuova Guida alla Comrpensione del Testo (De Beni et al., 2003)-, que fuera posteriormente adaptada al español por Abusamra y cols. (en prensa).

Abusamra, Cartoceti, Ferreres, De Beni y Cornorldi, siguiendo con el modelo de la comprensión de textos desde una perspectiva multicomponencial, presentan la adaptación al español de un test (Nuova guida alla comprensione del testo, De Beni et al., 2003) para evaluar la comprensión de textos. En el cual se obtienen datos normativos para niños de 9 a 12 años. El test Leer para Comprender (Abusamra et al., en prensa) toma como base un modelo global de once componentes: esquema básico del texto, hechos y secuencias, semántica léxica, estructura sintáctica, cohesión textual, inferencias, intuición del texto, jerarquía del texto, modelos mentales, flexibilidad y errores e incongruencias.

La sección Conferencias, Comunicaciones y Revisiones recoge tres trabajos de indudable interés académico y profesional:

En el primero, se presenta un trabajo de revisión sobre algunas de las investigaciones psicológicas y psicopedagógicas sobre trastornos del aprendizaje, en distintos países de Sudamérica, donde los Dres. Bravo-Valdivieso, Eslava, MilicicMuller, Mejía y quien coordina el monográfico, nos centramos en realizar una recorrida por algunos de los aportes relevantes en la materia en Argentina, Chile, Colombia, Paraguay, Perú y Uruguay. Su objetivo es mostrar una visión histórica de las distintas estrategias que ha elaborado cada uno de los países estudiados.

Por su parte la Dra. González, Guerra, Prato y Barrera, en su comunicación, aportan elementos de discusión respecto a un tema que es relevante al objetivo de este Monográfico, analizando los deberes escolares en el marco de las relaciones familia-escuela.

En última instancia, se presenta un estudio de revisión a cargo del Dr. Dansilio, sobre las bases anátomofuncionales de la dislexia, tema de real importancia y que vincula y pone de manifiesto el aporte de la Neuropsicología al estudio de los procesos lectores y sus alteraciones.
Este monográfico también se acompaña de la presentación de una serie de pruebas, de uso frecuente y necesario en las áreas en que se centran nuestros estudios.

Tenemos la ilusión de que este trabajo mancomunado de expertos de muy diversos lugares, sea una contribución al avance del conocimiento, la evaluación y tratamiento de los problemas que los niños padecen por su calidad de malos lectores.

\section{REFERENCIAS}

Carballo, M.; Cuadro, A. (2007). La velocidad de nominación en el déficit lector. IX Congreso Latinoamericano para el desarrollo de la lectura y la escritura. Montevideo, p. 115-123.

Cuadro, A. y Marin, J. (2007). Subtipos de lectores retrasados en español. Ciencias Psicológicas, v. 1 (2), p. 133-148.

Cuadro, A. (2006). Caracterización de los lectores retrasados. Memorias de las XIII Jornadas de Investigación UBA, v. II, p. 192-195.

Cuadro, A., Trías, D., y Castro, C. (2007). Ayudando a futuros lectores. Montevideo: Prensa Médica.

Cuadro, A. y Almansa, X. (2008). Caracterización de los lectores sordos: un estudio comparativo con los lectores normales oyentes. Memorias de la XII Jornadas de Investigación de la Facultad de Psicología- UBA, v. II, p. 319-321.

Cuadro, A. y Piñeyro, V. (2008). Caracterización de los lectores Braille: un estudio comparativo con buenos lectores videntes. Memorias de la XII Jornadas de Investigación de la Facultad de Psicología- UBA, v. II, p. 321-326.

Cuadro, A. y Trías, D. (2008). Desarrollo de la conciencia fonémica: evaluación de un programa de intervención. Revista Argentina de Neuropsicología, v 11, p.1-8.

Cuadro, A., Costa, D., Trias, D., y Ponce de León, P. (2009). Evaluación del Nivel Lector. Manual Técnico del Test de Eficacia Lectora (TECLE) de J. Marin y M. Carrillo. Montevideo: Prensa Médica.

Cuadro, A., Ilunday, A., y Puig, A. (2009). Habilidades prelectoras de niños en situación de pobreza. Memorias de la XIII Jornadas de Investigación de la Facultad de Psicología - UBA, v. II, p. 321-326.

Marin, J., Aveledo, F., Pagán, A., y Cuadro, A. (2007). ¿Por qué es más complicado distinguir entre "berenjena" y "berengena" que entre "escabeche" $y$ "escaveche"? Paper presentado en el XXV Congreso Internacional de AESLA, Murcia.

Marín, J., Pagán, A., Cuadro, A., y Aveledo, F. (2007). Adquisición de información ortográfica y mecanismo de recodificación fonológica: nuevas implicancias para el modelo de autoaprendizaje (Share, 1995). Paper presentado en el XXV Congreso Internacional de AESLA, Murcia.

Marin, J., Cuadro, A., y Pagán, J. (2007). Léxico Ortográfico y competencia lectora. Ciencias Psicológicas, 1 (1), p. 15-26. 